\title{
Editorial: Geospace Observation of Natural Hazards
}

\author{
Dimitar Ouzounov $^{1 *}$, Jann-Yenq Liu ${ }^{2}$, Patrick T. Taylor ${ }^{3}$ and Katsumi Hattori ${ }^{4}$ \\ ${ }^{1}$ Center of Excellence for Earth Systems Science and Observations, Chapman University, Orange, CA, United States, ${ }^{2}$ Center for \\ Astronautical Physics and Engineering, National Central University, Jungli City, Taiwan, ${ }^{3}$ Goddard Space Flight Center, National \\ Aeronautics and Space Administration, Greenbelt, MD, United States, ${ }^{4}$ Department of Earth Sciences, Chiba University, Chiba, \\ Japan
}

Keywords: geospace, natural hazards, satellite, earthquakes, flood, LEO, GPS/TEC, LAIC

\section{Editorial on the Research Topic}

\section{Geospace Observation of Natural Hazards}

This collection of technical papers aims to bring recent data from many sources into the study of natural hazards. They represent a multi-instrumental approach using both ground observations: Global Navigation Satellite System (GNSS); and Low Earth Orbiting Electromagnetic (LEO EM) satellites missions together with Earth Observations (EO), which could reveal new information. Results from latest satellite missions, [(NPP/NASA/NOAA(US), CENTINEL, Swarm/ESA (EU), HIMAWARI (JMA, Japan), FORMOSAT-5 (Taiwan, August 2017), CSES1 (China/Italy, Feb 2018), and FORMOSAT-7/COSMIC-2 (Taiwan/United States, May 2019)], are represented in this volume.

In addition, these results expand the analysis of assessing natural hazards using the latest geospace observations and by presenting the latest results with cross-disciplinary studies of earthquakes, volcanoes, tsunamis, and hurricanes/typhoons. These significant results advance existing interdisciplinary studies of several processes: for example, the lithosphere-atmosphereionosphere coupling processes.

Data from LEO satellites provide a comprehensive, global view of the variability of near-Earth space and complement ground-based observations that lack local coverage. These observations follow the earlier DEMETER (CNES, 2004-2010) satellite mission, specifically designed to make measurements in the thermosphere-ionosphere to investigate ionospheric anomalies and relate them to geohazards and space weather. We are taking advantage of the broad scope of observable electromagnetic activities by integrating ground-based observations and LEO satellites, helping to clarify the missing scientific knowledge in studying the genesis and evolution of the significant natural hazard events from space. All the studies presented are covered within the scope of Research Topics represented by two reviews, one brief research report, and nine original research papers and carried out by the community of international experts from 15 countries working in Geospace and natural hazards studies and reviewed by 28 peers, to whom we are graciously thankful.

Since the late seventies, many space observations have recorded signals associated with earthquakes. The DEMETER mission has constituted a milestone for space-based investigations of seismo-associated phenomena. A critical review of space-based observations covering a wide range of observations from electromagnetic field components (in a large band of frequencies) to plasmas parameters and from particles detection to thermal anomalies were summarized by (Picozza et al.). Along with a historical review, there is an assessment of the latest developments of the most recent mission investigating the near-Earth electromagnetic environment-CSES-01 satellite-developed within a Sino-Italian and Austrian collaboration. The second review paper (Conti et al.) provided a detailed summary of the observations carried out on the ground to identify pre-earthquake activity by distinguishing them from the background characterized by natural electromagnetic and artificial 
sources. This study focuses only on case studies and statistical analyses and the main hypotheses and models proposed in the literature to explain these observed phenomena.

The data from Seismic Electromagnetic Emissions associated with large earthquakes and the satellites DEMETER (Zhima et al.), Swarm (Zhu et al.), and NOAA Electron Bursts (Fidani) satellites provide new information about the seismicionospheric disturbance coupling mechanism. Spatialtemporal microwave brightness variations recorded from the Aqua satellite AMSR-2 show temperature (MBT) anomalies associated with the Mw 7.3 earthquake near the Iran-Iraq border on November 12, 2017 (Ding et al.). Transient variations in the atmosphere/ionosphere were recorded from data during Nepal's M7.8 and M7.3 earthquakes. They have also been studied with data from the NOAA Longwave radiation sensors and simultaneously with GPS/TEC and VLF/LF (Ouzounov et al. ). These data followed a general temporal-spatial evolutionary pattern within a large area (but inside the preparation region estimated by Dobrovolsky-Bowman); this feature has also been seen in other large earthquakes worldwide.

A complex model describing the ionosphere Sounding for Identification of Pre-seismic Anomalies was given by (Pulinets et al.). A physical mechanism explaining the ionospheric preseismic anomalies generated from the ground to the ionosphere were formulated within the framework of the LithosphereAtmosphere-Ionosphere Coupling (LAIC) model. The detailed classification of these anomalies was presented for different ionosphere regions, and the signatures of these pre-seismic anomalies were detected from the ground. Satellite-based instruments were described and classified, defining the methodology of the precursor's identification from ionospheric multi-instrumental measurements.

A new analysis of sub-ULF $(<1 \mathrm{msec})$ magnetic field measurements made during the time of the Chi-Chi earthquake, Taiwan, September 20, 1999, by a joint investigation of satellite measurements in interplanetary space (ACE satellite) and on-ground measurements were made (Anagnostopoulos). Four groups of sub-ULF waves in the Taiwan data coincided, in time, with the quasi-periodic detection of two solar wind streams by the ACE satellite with approximately the solar rotation period ( 28 days). This observational evidence shows that sub-ULF electromagnetic radiation on the Earth was probably part of geotectonic processes formulating for the Taiwan 1999 earthquake.

The propagation of sub ionospheric VLF/LF $(15-50 \mathrm{kHz})$ frequencies from navigation or time service transmitters over distances of thousands of kilometers (with low attenuation $\sim 2-3 \mathrm{~dB}$ per $\mathrm{Mm}$ ) enables remote sensing over large regions of the upper atmosphere in which ionospheric modifications lead to changes in the received amplitude and phase of the signals. Such results were included in the Research volume reported for the inland earthquake in Japan (Nagao et al.) and before and during the 2015 M7.8 and M7.3 Gorkha-Nepal earthquakes (Ouzounov et al.). Nagao et al. introduced an electromagnetic-wave arrival discrimination algorithm that combines the autoregressive model and the Akaike information criterion to obtain accurate data on the time of arrival (TOA) of electromagnetic waves.

The seismo-ionospheric perturbations prior to large earthquakes are based on total electron content (TEC) in the global ionosphere map is quite challenging since it is due to the dynamic complexity of the ionosphere and the identification of precursory ionospheric changes. This study analyzed the total electron content (TEC) in the global ionosphere map, with a singular Spectrum Analysis of the TEC. Chen et al. investigate the GPS/TEC changes prior to $M \geq 6.0$ earthquakes in China from 1998 to 2013 to identify possible seismo-ionospheric precursors. They confirmed that the negative anomalies are dominant $1-5$ days before the earthquake at the fixed point $\left(35^{\circ} \mathrm{N}, 90^{\circ} \mathrm{E}\right)$ during 0600-1000 LT.

The Flood susceptibility modeling of central India's subtropical Middle Ganga Plain (MGP) has been presented with a machine learning ensemble approach (Pandey et al.) This study compares two machine learning ensemble models, one first time built and the other used in other natural hazards but not for floods, in mapping the flood susceptible zones in the subtropical fluvial basin of the MGP. The result indicates that both ensembles delineate flood susceptible zones in low-latitude, subtropical monsoonal regions like MGP with reasonably good accuracy and precision.

In conclusion, the availability of new global satellite data and models on the thermosphere/ionosphere interactions demonstrate that satellites that have not been specifically designed for natural hazard studies can provide valuable contributions to this field. Such multi-instrumental observations could expand our knowledge of the geosphere's interaction associated with natural hazards.

\section{AUTHOR CONTRIBUTIONS}

DO organized and wrote the manuscript. PT, J-YL, and $\mathrm{KH}$ provided critical feedback. All authors contributed to the submitted version of the article.

Conflict of Interest: The authors declare that the research was conducted in the absence of any commercial or financial relationships that could be construed as a potential conflict of interest.

Publisher's Note: All claims expressed in this article are solely those of the authors and do not necessarily represent those of their affiliated organizations, or those of the publisher, the editors and the reviewers. Any product that may be evaluated in this article, or claim that may be made by its manufacturer, is not guaranteed or endorsed by the publisher.

Copyright (c) 2022 Ouzounov, Liu, Taylor and Hattori. This is an open-access article distributed under the terms of the Creative Commons Attribution License (CC BY). The use, distribution or reproduction in other forums is permitted, provided the original author(s) and the copyright owner(s) are credited and that the original publication in this journal is cited, in accordance with accepted academic practice. No use, distribution or reproduction is permitted which does not comply with these terms. 